\begin{tabular}{|c|c|}
\hline \multirow{3}{*}{ 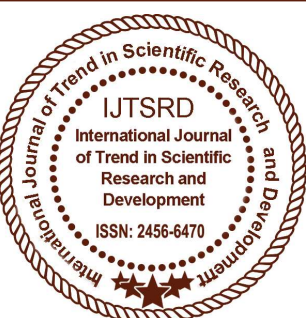 } & $\begin{array}{l}\text { International Journal of Trend in Scientific } \\
\text { Research and Development (IJTSRD) }\end{array}$ \\
\hline & International 0 \\
\hline & ISSN No: 2456 - 6470 | www.ijtsrd.com | Volume - 2 | Issue - 5 \\
\hline
\end{tabular}

\title{
Comparative Analysis of Particle Swarm Optimization Based Routing Protocols for MANET and VANET
}

\author{
Geetika Sharma, Anupam Mittal \\ Assistant Professor, \\ Chandigarh University, Ludhiana, Punjab, India
}

\begin{abstract}
Swarm Intelligence is an artificial intelligence discipline which is concerned with the design of intelligent multi-agent systems by taking inspiration from the collective behaviours of social insects and other animal societies. PSO is a population based optimization technique use for finding optimum solution. PSO technique is originated from social behavior bird flocking. The purpose of the research is to compare particle swarm optimization based routing algorithms used in MANET and VANET. ISwarm intelligence based routing algorithm will more promising for specific nature of ad- hoc networks scenarios/constraints/environmental conditions and will tune and simulate to get an efficient and effective routing protocol for MANET and VANET.
\end{abstract}

Keywords: MANET, VANET, honeybee, Particle swarm optimization, Swarm Intelligence, Ant Colony Optimization (ACO).

\section{INTRODUCTION}

Ad-hoc networks [1] communicate over wireless media between stations directly in a peer to peer fashion without the help of wired base station or access points. Mobile Ad Hoc Networks (MANETs) is one of ad-hoc network communication system which is extensively used. MANETs are built up of a collection of mobile nodes which have no fixed infrastructure. The nodes communicate independently through wireless network without any central control. Routing is the task of directing data packets from a source node to a given destination. The routing is complex procedure due to the dynamic topology, limited process, storing capability, bandwidth constraints and lack of the central control.
Routing protocols for MANETs suffer from certain inherent shortcomings. First the proactive routing schemes used in MANET like Destination Sequenced Distance Vector (DSDV) continuously updates the routing tables of mobile nodes which large portion of the scarce network capacity for exchanging huge chunk of routing table data. This reduces the available capacity of the network for actual data communication. Secondly the on-demand routing protocols like Ad-Hoc On-Demand Distance Vector (AODV) and Dynamic Source (DSR) routing. These protocols launch route discovery, and require the actual communication to be delayed until the route is determined. This is not suitable for real-time data and multimedia communication applications.

Swarm Intelligence (SI) is the collective behaviour of decentralized, self-organized systems, natural or artificial in biological systems. SI is an appealing solution when routing becomes a crucial problem in a complex network scenario, where traditional routing techniques fail completely. SI systems are typically made up of a population of simple agents interacting locally with one another and their environment.

Particle swarm optimization (PSO) [9] is a robust stochastic optimization technique based on the movement and intelligence of swarms. PSO applies the concept of social interaction to problem solving. It uses a number of agents (particles) that constitute a swarm moving around in the search space looking for the best solution. Each particle is treated as a point in an N-dimensional space which adjusts its "flying" according to its own flying experience as well as the flying experience of other particles. 
Routing algorithms which are based on swarm intelligence are:-

$>$ Collective system capable of accomplishing difficult tasks in dynamic and varied environments without any external guidance or control and with no central coordination.

Achieving a collective performance which could not normally be achieved by an individual acting alone.

> Constituting a natural model particularly suited to distribute problem solving.

\section{SWARM INTELLIGENCE}

Swarm Intelligence (SI) is mainly defined as the behaviour of natural or artificial self-organized, decentralized systems. Swarms interact locally with each other or with external agents i.e. environment and can be in the form of bird flocks, ants, bees etc. It is the property of a system whereby the collective behaviour of (unsophisticated) agents interacting locally with their environment cause coherent functional global patterns to emerge. SI provides a basis with which it is possible to explore collective (or distributed) problem solving without centralized control or the provision of a global model.

SI based approaches are nature and bio-inspired. Swarms are abundantly found in nature. In the nature animals form into swarms to search food, build nests, to hunt and avoid being hunted etc. Each individual swarm has simple rule of access to a limited amount of information via its immediate neighbours or local environment. It consists of mainly PSO, Ant colony optimization (ACO) and honeybee paradigms. The population of the potential solution is called as swarm and each individual in the swarm is defined as particle. The particles fly in the swarm to search their best solution based on experience of their own and the other particles of the same swarm.

The SI based approaches are more promising from other conventional techniques for optimization problems, due to the nature, architecture, topology and functionality of ad hoc networks. SI approaches are more suitable for the routing and energy resources optimization related issues in MANETs.

Bio inspired, SI approaches are more promising for Ad-hoc networks due to the following prominent aspects 1.locality of interactions 2.availability of multiple paths 3. Self organising 4. Failure backup 5 . ability to adapt in a quick and robust way to topological and traffic changes and component failures.

\section{Particle Swarm Optimization}

Particle swarm optimization (PSO) is a populationbased stochastic optimization technique developed by Kennedy and Eberhart in 1995. The use of PSO algorithm is to determine optimum solution. In PSO algorithm, an optimal solution is found from the social behavior of bird flocking. With the aim of discovering patterns that govern the ability of birds to fly synchronously and to suddenly change direction with a regrouping in an optimal formation. PSO consist of group of individual called as -particlesll. The particles fly through multidimensional search space looking for best solution. The effective solution can be obtained by using common information of the group and information own by particles itself. For better performance, each particle adjusts its velocity time to time based on its current velocity with respect to its previous best position and also the position of current best particle in the population. For solving the optimization problems and combinatorial problems PSO algorithm is most useful [10].

A PSO algorithm maintains a swarm of particle represents the potential solution. In simple terms, particles are randomly fly through the multidimensional search space, the particle adjust its position according to its own experienced and that of its neighbors. Let $x i(t)$ represent the position of particle $i$ in the search space at a discrete time step $t$. When the particle moves it get some velocity and its original position is changed, i.e. It is the velocity vector that drives the optimization process and reflects both the related knowledge of the particle and socially exchanged information from the particle's neighborhood. The experimental knowledge of particle is directly proportional to the distance of particle from its own best position known as particle best (pbest). There are basically two concept of PSO algorithm, namely pbest and gbest. In global best (gbest) the neighborhood for each particle is entire swarm. The network establish in gbest PSO is by using star topology, in which the social particle velocity is updated regularly which gives information obtained from all the particle in swarm.

In personal best (pbest) PSO the network form by using ring social network topology where smaller 
neighborhood are define for each particle. In this network, information exchanged between the neighborhoods of particle, which provide local knowledge of the environment.

\section{SWARM INTELLIGENCE ROUTING ALGORITHM}

Routing protocols are proposed for mobile ad-hoc networks have revealed that the on-demand routing protocols perform better in terms of packet delivery and routing overhead than proactive routing schemes especially in the presence of node mobility. Proactive and hybrid schemes do not perform well in dynamic topologies because of the following two major factors:

1.Slow detection of broken links and 2. Periodic exchange of route updates even when routes are not needed. [2]

The routing protocols under idealistic settings where all nodes function properly. The behaviour of routing protocols where some nodes may be faulty [3]. The paper has proposed that AODV outperforms OLSR in terms of packet delivery ratio while OLSR achieves a much lower end to end delay.

A new cooperative coevolving particle swarm optimization (CCPSO) [4] algorithm addresses the issue of scaling up particle swarm optimization (PSO) algorithms in solving large-scale optimization problems (up to 2000 real-valued variables). The proposed CCPSO2 builds on the success of an early CCPSO that employs an effective variable grouping technique random grouping. CCPSO2 adopts a new PSO position update rule that relies on Cauchy and Gaussian distributions to sample new points in the search space, and a scheme to dynamically determine the coevolving subcomponent sizes of the variables. Results and analysis suggest that CCPSO2 is a highly competitive optimization algorithm for solving largescale and complex multimodal optimization problems.

The designing of optical networks [5] are focused on the wavelength division multiplexing (WDM) technology. This technology divides the huge bandwidth of an optical fiber into different wavelengths, providing different available channels per link of fiber. However, when it is necessary to establish a set of demands, a problem comes up. This problem is known as a routing and wavelength assignment (RWA) problem. Depending on the traffic pattern, two varieties of a RWA problem have been considered in the literature: static and dynamic. Swarm intelligence is very suitable to solve the RWA problem, and presumably that it may obtain such quality results not only in diverse telecommunication optimization problems, but also in other engineering optimization problems.

A new proposal of using particle swarm optimization algorithms to solve multi-objective optimization problems is presented. The algorithm is constructed based on the concept of Pareto dominance, as well as a state-of-the-art 'parallel' computing technique that intends to improve algorithmic effectiveness and efficiency simultaneously. The proposed parallel particle swarm multi-objective evolutionary algorithm (PPS-MOEA) [9] is tested through a variety of standard test functions its performance is compared with six noted multi-objective algorithms. The computational experience gained from the first two experiments indicates that the algorithm proposed in this article is extremely competitive when compared with other MOEAs, being able to accurately, reliably and robustly.

Inspired by swarm intelligence observed in social species, the artificial self-organized networking (SON) [10] systems are expected to exhibit some intelligent features (e.g., flexibility, robustness, decentralized control, and self-evolution, etc.) It is concluded that the bio-inspired mechanism is indeed a powerful source of innovative networking paradigm for artificial SON systems.

Swarm intelligence [7] a new approach for an on demand ad-hoc routing algorithm is proposed. The key components of feedback mean that an individual recruits other individuals by some directive, such as dancing of bees in order to lead some other bees onto a specific food source site. Feedback, fluctuations and multiple interactions [17]. Positive Negative feedback avoids all individuals accumulating on the same task by rebalancing the attraction negatively, such as abandoning the exhausted food source. 3

\section{CONCLUSION}

MANET consists of a set of mobile nodes and challenges in routing due to dynamic network topology, power conservation in wireless nodes. The characteristics of MANET such as bandwidth constrained, energy constrained, limited physical security and dynamic network topology etc. 
Randomized change of domain causes change in topology of networks. Hence the major issue related to MANETs is routing. The proactive and reactive routing algorithms have their own limitations and are not found suitable for routing in MANET mainly when real time applications. The aim of the study is to investigate the advantages of the different approaches in relation to the characteristics of urban environments and to concrete application models for real-world MANETS.

\section{REFERENCES}

1. Siva. R. M, C. and B. S. Manoj, "Ad-Hoc Wireless Networks: Architecture and Protocols", Pearson, USA, 2004.

2. E. Royer and C. K. Toh, "A review of current routing protocols for ad-hoc mobile wireless networks", IEEE Personal Communications, 1999.

3. Rajagopalan. R, Dahlstrom, "Performance comparison of routing protocols in mobile ad hoc networks in the presence of faulty nodes", \{IEEE International Conference On Wireless Information Technology And Systems (ICWITS)\}, 11-16 NOV. 2012.

4. Xiaodong Li, Xin Yao, "Cooperatively Coevolving Particle Swarms for Large Scale Optimization", \{IEEE Transactions On Evolutionary Computation $\}$, VOL. 16, NO. 2, APRIL 2012.

5. Rubio largo A, Vega Rodriguez M, Gomez Pulido, Sanchez-Perez, "Comparative Study on Multi objective Swarm Intelligence for the Routing and Wavelength Assignment problem", \{ IEEE Transactions On Systems, Man And Cybernetics Part C , Volume:42, Issue: 6 , Nov 2012.

6. Asadinia. S, Rafsanjani. M. k, Saeid. A. B, "A novel routing algorithm based-on ant colony in Mobile Ad hoc Networks", \{ 3rd IEEE International Conference on Ubi-media Computing (U-Media)\}, 5-6 July 2010.

7. G. Di Caro, F. Ducatelle, L. M. Gambardella, "Swarm Intelligence for routing in mobile ad hoc networks", \{ In Proceedings of the 2005 IEEE Swarm Intelligence Symposium, SIS 2005\}, pp. 76-83, 2005.
8. B. Chandra Mohan \& R. Baskaran, "Survey on Recent Research and Implementation of Ant Colony Optimization in Various Engineering Applications", \{International Journal of Computational Intelligence Systems Taylor and Franchis\}, Volume 4, Issue 4, 2011.

9. Shu-Kai S. Fana \& Ju-Ming Changa, "A parallel particle swarm optimization algorithm for multi-objective optimization problems", \{Engineering Optimization Taylor and Franchis\}, Volume 41, Issue 7, 2009.

10. Zhang, Z. ; Long, K. ; Wang, J. ; Dressler, F ,“On Swarm Intelligence Inspired Self-Organized Networking: Its Bionic Mechanisms, Designing Principles and Optimization Approaches", Communications Surveys \& Tutorials, IEEE, ( Issue: 99 ), 04 July 2013.

11. Bahriye Akay, Dervis Karaboga, "A modified Artificial Bee Colony algorithm for real-parameter optimization", \{ Elsevier\}, 2010.

12. F. Ducatelle, G. D. Caro, and L. M. Gambardella, "Using ant agents to combine reactive and proactive strategies for routing in mobile ad hoc networks", \{International Journal of Computational Intelligence and Applications\}, vol. 5, no. 2, pp. 169-184, 2005.

13. F. Dressler and O. Akan, "Bio-inspired networking: from theory to practice", $\{$ IEEE Communication Magazine. \}, vol. 48, no. 11, pp. $176-183,2010$

14. F. Dressler and O. Akan, "A Survey on Bioinspired Networking", \{Elsevier Computer Networks\}, vol. 54, no. 6, pp. 881-900, 2010.

15. O. Tonguz, "Biologically inspired solutions to fundamental transportation problems", \{IEEE Communication. Magazine \}, vol. 49, no. 11, pp. 106-115, Nov. 2011.

16. Frederick Ducatelle, Gianni A. Di Caro, Luca M. Gambardella," An Evaluation of Two Swarm Intelligence MANET Routing Algorithms in an Urban Environment", \{IEEE Swarm Intelligence Symposium $\}, 2010$. 
International Journal of Trend in Scientific Research and Development (IJTSRD) ISSN: 2456-6470

17. Roth, M., "Termite: A Swarm Intelligent Routing Algorithm for Mobile Wireless Ad-Hoc Networks", \{Thesis and Dissertations\}, Cornell University Graduate School (2005).

20. Falko Dresser, "A Study of Self Organization Mechanism in Ad-hoc and Sensor Networks", \{Elsevier Computer Communications \}, Vol. 31(13), 2008.

18. Ajith Abraham, Crina Grosan, Vitorino Ramos, "Stigmergic Computational Intelligence", \{Spinger\}, 2010.

19. Muhammad Saleem a, Gianni A. Di Caro b, Muddassar Farooq, "Swarm intelligence based routing protocol for wireless sensor networks: Survey and future directions", \{Information Sciences Elsevier\}, 2010.

21. S. Lalwani, S. Singhal, R. Kumar and N. Gupta, "A Comprehensive Survey: Applications of MultiObjective Particle Swarm Optimization (Mopso) Algorithm", \{Transactions on Combinatorics\}, Vol. 2 No. 1 (2013), pp. 39-101.

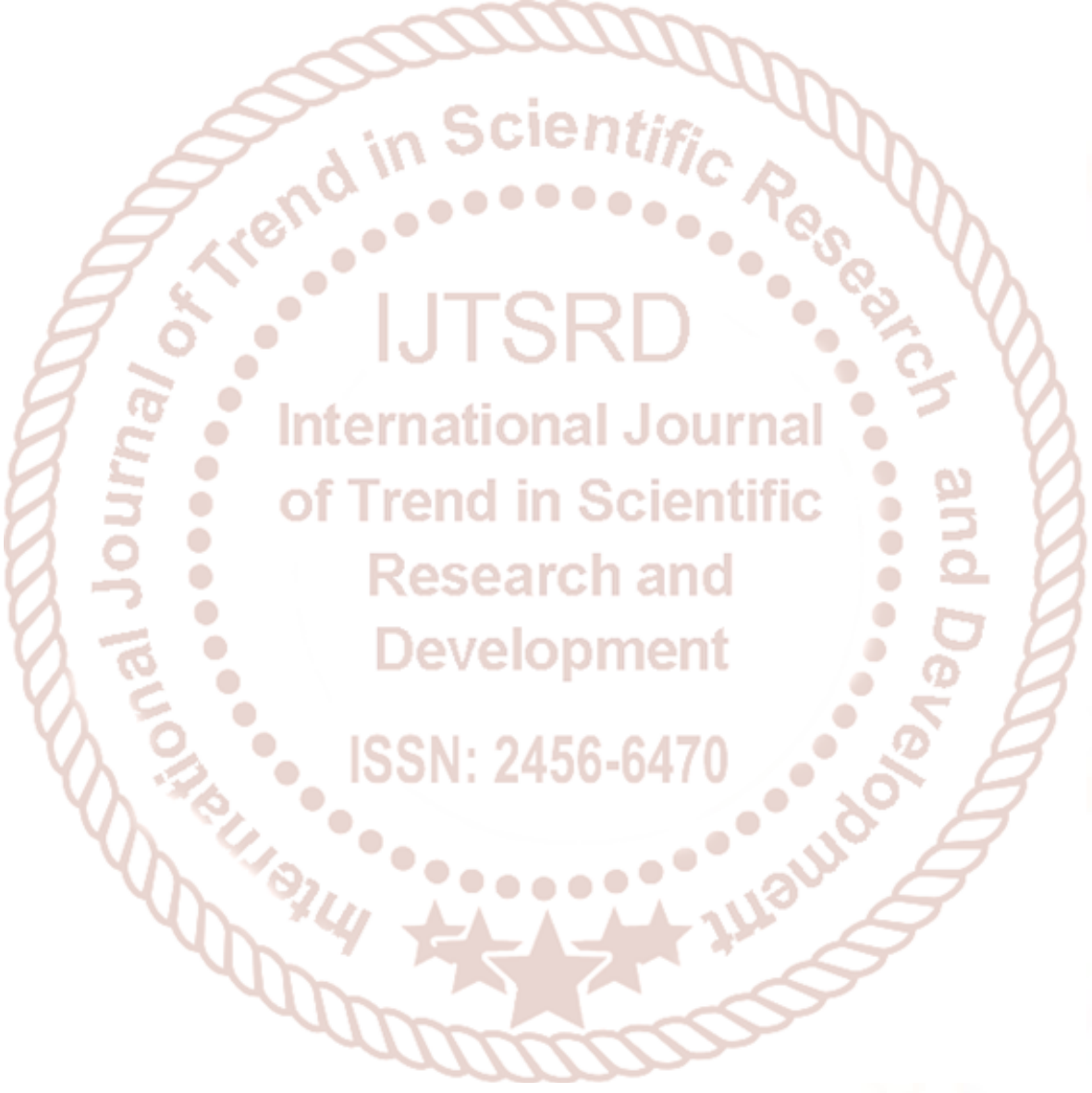

